\title{
Médiévales
}

Langues, Textes, Histoire

80 | printemps 2021

Animaux marins

\section{Beatrice BORGHI, Il Mediterraneo di Anselmo Adorno. Una testimonianza di pellegrinaggio del tardo medioevo}

Bologne, Pàtron Editore, 2019, 560 p.

Térence Le Deschault de Monredon et Beatrice Borghi

\section{OpenEdition}

\section{Journals}

Édition électronique

URL : https://journals.openedition.org/medievales/11604

DOI : 10.4000/medievales. 11604

ISSN : 1777-5892

\section{Éditeur}

Presses universitaires de Vincennes

Édition imprimée

Date de publication : 8 juillet 2021

Pagination : 192-194

ISBN : 978-2-37924-182-6

ISSN : 0751-2708

\section{Référence électronique}

Térence Le Deschault de Monredon et Beatrice Borghi, « Beatrice BoRGHI, II Mediterraneo di Anselmo

Adorno. Una testimonianza di pellegrinaggio del tardo medioevo », Médiévales [En ligne], 80 |

printemps 2021, mis en ligne le 14 septembre 2021, consulté le 03 janvier 2023. URL : http://

journals.openedition.org/medievales/11604; DOI : https://doi.org/10.4000/medievales.11604 
introduction dense et un glossaire des termes gallois ; il s'achève avec une bibliographie (particulièrement développée pour les éditions de textes et les traductions) et un index détaillé. Notons enfin que tous les textes cités en langue originale sont pourvus d'une traduction en anglais : malgré la technicité de certains passages, le lecteur ignorant des langues celtiques n'est presque jamais incommodé, ce qui constitue un véritable tour de force.

Alban GAUTIER (Université de Caen Normandie)

\section{Beatrice Borghi, Il Mediterraneo di Anselmo Adorno. Una testimonianza di pellegrinaggio del tardo medioevo, Bologne, Pàtron Editore, 2019, $560 \mathrm{p}$.}

Le plus ancien témoignage de pèlerinage que nous connaissions remonte au IV siècle. Il s'agit de la relation de voyage de l'anonyme pèlerin de Bordeaux qui se rendit à Jérusalem. Depuis, de nombreux auteurs ont livré soit leur récit de pèlerinage, soit des conseils pour guider les pèlerins vers les lieux saints, que ce soit Jérusalem, Rome ou Compostelle. Ce type de littérature, dont le fameux livre V du Liber Sancti Jacobi est l'un des fleurons, s'est surtout épanoui à la fin du Moyen Âge.

Ainsi, nous avons conservé la très riche relation de voyage décrivant le périple d'Anselmo Adorno, marchand italien parti de Bruges avec son fils Jean en 1470 pour un long pèlerinage à Jérusalem. Ce récit est connu par trois manuscrits seulement. En réalité, aucun des trois n'est le manuscrit original, rédigé par Giovanni Adorno, ou la copie remise au roi d'Écosse auquel l'œuvre est dédiée. Le manuscrit 330 de la Bibliothèque municipale de Lille est une copie légèrement abrégée du texte intégral, composé quelques décennies après le retour à Bruges, à la fin du $x^{\mathrm{e}}$ siècle. Un deuxième manuscrit remontant au $\mathrm{XIX}^{\mathrm{e}}$ siècle s'avère être une copie d'un manuscrit disparu qui correspondait à une version plus ancienne et plus longue, sans doute très proche du texte original offert à Jacques III d'Écosse. Il s'agit du manuscrit M1 24 de la Bibliothèque Vauban de 1'Université catholique de Lille. Enfin, en 1491, Romboudt de Doppere réalisa une traduction en flamand, fortement abrégée, du texte latin de Giovanni Adorno, sous la forme d'un guide pour les pèlerins en Terre sainte, complété par d'autres informations utiles à l'entreprise d'une telle expédition. Cette version fut éditée en 1893 par E. Feys dans les Annales de la Société d'émulation de Bruges.

Le contenu du manuscrit 330 de la Bibliothèque municipale de Lille n'est pas inédit, puisque, dès 1978, Jacques Heers et Georgette de Groër en avaient proposé la première édition et une traduction intégrale en français. Cette unique édition et traduction de l'ensemble de cet important témoignage méritait une nouvelle approche critique et c'est ce que nous offre Beatrice Borghi, historienne de l'Université de Bologne, en publiant une édition révisée du texte latin, accompagnée d'une traduction inédite en italien. Quant aux descriptions supplémentaires propres au manuscrit M1 24 de la bibliothèque de l'Université catholique de Lille qui figuraient en appendice dans l'édition de J. Heers et G. de Groër, elles sont ici intégrées dans le corps du texte, en italiques, certainement dans une volonté de se rapprocher le plus possible de la version originale perdue. Le résultat rend la lecture des différentes péripéties du voyage facile et sans solution de heurt.

Dans l'édition française, le choix des auteurs avait été de rédiger une courte introduction (une vingtaine de pages), mais d'accompagner le texte de nombreuses notes, parfois assez longues. Le parti pris par Beatrice Borghi est tout autre, puisqu'elle a préféré réduire les notes au strict nécessaire et produire une riche première partie (131 pages) qui va bien au-delà d'une simple introduction. Le résultat de ce choix se manifeste, là aussi, par une meilleure fluidité de lecture.

Après une préface de Véronique de Limburg Stirum, descendante de la famille Adorno, et une introduction de Laura Galoppini, spécialiste des marchands italiens installés à Bruges à la 
fin du Moyen Âge, la première partie du livre est composée de huit chapitres. Le premier est une explication du contexte, historique, sociologique et familial, dans lequel Anselmo Adorno décida d'entreprendre son pèlerinage en Terre sainte. Les sept autres chapitres glosent les différentes observations d'Anselmo durant son voyage et apportent une riche documentation permettant de mieux apprécier ce que pouvait représenter une telle aventure.

Avant d'entamer la seconde partie du livre, composée de l'édition du texte latin et de sa traduction italienne en regard, Beatrice Borghi a eu l'heureuse idée d'insérer une reproduction photographique de l'intégralité du ms. 330 de la BM de Lille, au tiers de sa grandeur. Le résultat est à peu près illisible à l'œil nu, mais la bonne qualité des images offre toutefois à ceux qui le désireraient la possibilité de vérifier, à condition de se munir d'une loupe, certains points de l'édition. Cela permet également d'admirer la belle mise en page du manuscrit, la régularité de l'écriture et quelques curiosités, comme ce tableau de correspondances entre les lettres de l'alphabet arabe et les syllabes qu'elles expriment, ou encore ce dessin d'une pièce de monnaie en circulation en Égypte en 1470.

Le volume est accompagné d'une riche bibliographie, intelligemment scindée en deux parties : la première, thématique, donne des indications sur les pèlerins et pèlerinages à la fin du Moyen Âge, sur le pèlerinage en Terre sainte et sur la famille Adorno, tandis que la seconde ne concerne que les ouvrages cités en notes. Deux index (lieux et personnes), bien plus fournis que dans l'édition française de 1978, complètent l'ensemble.

Les Adorno sont une famille de marchands génois établis à Bruges depuis trois générations au moment où Anselmo et son fils Giovanni entreprennent leur périple vers la Terre sainte. En réalité, plus qu'un simple marchand, Anselmo est un véritable aristocrate. Il se qualifie de miles (chevalier), il détient des terres et joue un rôle politique et diplomatique important, à la fois auprès des ducs de Bourgogne et du roi d'Écosse, à qui le récit de son pèlerinage est dédié. Les Adorno de Bruges sont des gens cultivés : le père d'Anselmo, Pietro, fonda par testament la première bibliothèque publique des Pays-Bas méridionaux en précisant qu'après sa mort, sa collection privée serait accessible à tous dans la chapelle familiale de Jérusalem à Bruges. Il s'agit donc d'une famille enrichie dans les affaires, lettrée et possédant une culture chevaleresque (Anselmo participe et gagne plusieurs tournois dans sa jeunesse). Anselmo parachève sa carrière politique en étant nommé seigneur de Corthuy par le roi d'Écosse et en réalisant des missions diplomatiques pour Philippe le Bon et Charles le Téméraire, en Italie et au Proche-Orient.

Dans son étude liminaire, outre ce qui vient d'être dit sur la famille Adorno, Beatrice Borghi rappelle quelques faits importants, comme la curiosité quasi « touristique » qui, en sus de leur motivation religieuse, meut les pèlerins en cette fin du Moyen Âge, ou encore la présence féminine dans ces longs périples outre-Méditerranée, tout en précisant que les récits de pèlerins s'attachent davantage à la description des femmes orientales à l'apparence exotique. Elle souligne également le penchant familial des Adorno pour les merveilles de la Terre sainte, avec l'aïeul Oppicino qui avait, dès 1269, entrepris le pèlerinage à Jérusalem avant de s'installer à Bruges, le père et l'oncle d'Anselmo, qui, eux aussi, firent le voyage outre-mer d'où ils rapportèrent l'idée d'édifier en 1435 à Bruges une chapelle construite sur le modèle du Saint-Sépulcre et dédiée à Jérusalem, puis enfin Adorno et son fils Giovanni. L'auteur décrit le changement de panorama géopolitique induit par la prise de Constantinople par les Turcs en 1453 et leur domination sur la Méditerranée, qui modifia sensiblement les conditions de navigation des Vénitiens et des Génois ainsi que les relations commerciales avec l'Orient. Le compte rendu de voyage d'Anselmo Adorno, avec la description des contrées infidèles et de leurs habitants, semble d'ailleurs avoir été offert à Jacques III d'Écosse pour l'aider à préparer une croisade soutenue par le pape Pie II. En témoigne notamment le fait qu'Anselmo Adorno n'a pas suivi les pèlerins qui embarquaient une ou deux fois l'an à Venise sur des bateaux affrétés 
par la cité et dont le but du voyage n'allait pas au-delà de Jaffa et Jérusalem. Au lieu de cela, Anselmo et Giovanni partent de Gênes sur un gros navire marchand et avec le désir de voir bien plus de régions que celles proposées par les « voyages organisés » vénitiens. C'est aussi ce qui rend leur témoignage particulièrement intéressant.

Le contenu du texte des Adorno a une grande valeur à plusieurs titres. Bien sûr, il décrit minutieusement les lieux saints visités et consacre plusieurs pages à inventorier tous les détails du Saint Sépulcre, ce qui constitue l'essence même d'un récit de pèlerinage. De plus, il dépeint une image de la Méditerranée à la fin du xv siècle, avec ses peuples, leur milieu de vie et leurs coutumes. Adorno partage, par exemple, son admiration pour l'utilisation des pigeons voyageurs qu'il constate dans le port d'Alexandrie. Cependant, cette image est teintée de toutes les croyances qu'un lettré occidental pouvait avoir sur l'Orient et c'est là un point tout à fait passionnant puisqu'il s'agit d'une sorte de miroir qui nous donne à voir le portrait psychologique de l'auteur et de son père. De sorte que, si on rencontre en Afrique des lions et des ours, on peut aussi y trouver, selon Adorno, des griffons. Quant aux femmes de Sardaigne, certaines d'entre elles ont dans les yeux des pierres précieuses qui peuvent tuer celui qu'elles regardent avec courroux !

Enfin, un certain nombre de considérations nous dévoile également un très intéressant panorama de la situation politique et stratégique en Méditerranée. Ainsi, la menace turque prend une place importante, et la résistance de l'île de Rhodes est considérée par Adorno comme la dernière barrière face à l'audace de ces ennemis prêts à tout pour conquérir l'Occident. Adorno décrit d'autres faits pouvant servir au roi d'Écosse dans le cadre d'une croisade, par exemple l'organisation des Mameluks sous le gouvernement du sultan, au Caire, ou l'aspect des ports et des fortifications qu'il croise sur son chemin.

Cette nouvelle présentation du récit de voyage d'Anselmo et Giovanni Adorno constitue un bel ouvrage, particulièrement utile pour se faire une idée de la situation du monde méditerranéen en cette fin du $\mathrm{xv}^{\mathrm{e}}$ siècle et pour voir comment la percevait un riche aristocrate lettré d'origine italienne rompu aux voyages à travers l'Europe. En définitive, Beatrice Borghi rappelle l'importance de ce texte pour la connaissance des rapports diplomatiques entre Orient et Occident et met à disposition du lecteur des informations détaillées permettant une bonne compréhension du contexte de rédaction de ce précieux témoignage.

Térence Le Deschault DE MONREDON (Conseiller pour le patrimoine de la commune de Theys) 